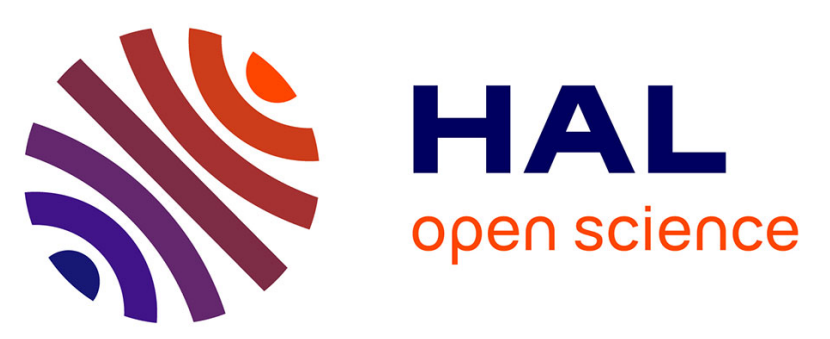

\title{
Estimation of the error on the mean velocity and on the Reynolds stress due to a misoriented ADV probe in the horizontal plane: case of experiments in a compound open-channel
}

\author{
Y. Peltier, N. Rivière, Sébastien Proust, Emmanuel Jean Marie Mignot, \\ André Paquier, K. Shiono
}

\section{To cite this version:}

Y. Peltier, N. Rivière, Sébastien Proust, Emmanuel Jean Marie Mignot, André Paquier, et al.. Estimation of the error on the mean velocity and on the Reynolds stress due to a misoriented ADV probe in the horizontal plane: case of experiments in a compound open-channel. Flow Measurement and Instrumentation, 2013, 34, p. 34 - p. 41. 10.1016/j.flowmeasinst.2013.08.002 . hal-00908959

\section{HAL Id: hal-00908959 \\ https://hal.science/hal-00908959}

Submitted on 25 Nov 2013

HAL is a multi-disciplinary open access archive for the deposit and dissemination of scientific research documents, whether they are published or not. The documents may come from teaching and research institutions in France or abroad, or from public or private research centers.
L'archive ouverte pluridisciplinaire HAL, est destinée au dépôt et à la diffusion de documents scientifiques de niveau recherche, publiés ou non, émanant des établissements d'enseignement et de recherche français ou étrangers, des laboratoires publics ou privés. 


\title{
Estimation of the error on the mean velocity and on the Reynolds stress due to a misoriented ADV probe in the horizontal plane: case of experiments in a compound open-channel
}

\author{
Y. Peltier ${ }^{\mathrm{a}, \mathrm{b}, *}$, N. Rivière ${ }^{\mathrm{a}}, \mathrm{S}$. Proust ${ }^{\mathrm{b}}$, E. Mignot ${ }^{\mathrm{a}}$, A. Paquier $^{\mathrm{b}}$, K. Shiono $^{\mathrm{c}}$ \\ ${ }^{a}$ Laboratoire de Mécanique des Fluides et Acoustique (LMFA, CNRS UMR5509, Université de Lyon) INSA de Lyon, Bât Jacquard, 20 av. A. \\ Einstein, 69621 Villeurbanne, France \\ ${ }^{b}$ Irstea, UR HHLY,Hydrology-Hydraulics, 5 rue de la Doua, CS 70077, 69626 Villeurbanne Cedex, France \\ ${ }^{c}$ Loughborough University, civil and building engineering department, Leicestershire, UKLE11 3TU, United Kingdom
}

\begin{abstract}
This study aims at evaluating the error on the horizontal components of the mean-velocity and of the Reynolds stress measurements due to a misorientation of an Acoustic Doppler Velocimeter (ADV) in the horizontal plane. The misorientation of the ADV is represented by a misalignment angle, which is the angle between the ADV coordinate frame and the reference frame. The error due to the misorientation is first analytically determined. Thereafter, using experiments in a compound channel, the error is evaluated based on an angle of $2.5^{\circ}$ representing the maximum possible angle of misalignment by human eyes adjustment. While the maximal error does not exceed 5\% for the longitudinal mean velocity and $14 \%$ for the normal Reynolds stresses, it can be larger than $100 \%$ for the lateral mean velocity and largely greater than $100 \%$ for the Reynolds shear stress. Such a degree of error appears to be similar to those worked out for classical flows from the literature with a mean velocity gradient mainly occurring in the horizontal plane (mixing layers, plane jets) or in the vertical plane (boundary layers). This error is finally compared with the uncertainty due to the various sources of noise that pertubate the turbulence measurement. This study reveals that they both are comparable, therefore indicating that the error due to the probe misorientation should not be neglected, as it is too often the case.
\end{abstract}

Keywords: ADV, error, uncertainty, misorientation, mean velocity, Reynolds stress, compound channel

\section{Introduction}

The Acoustic Doppler Velocimeter (ADV) is widely used in laboratory experiments and field works for measuring flow velocities. The existing commercial versions (field probes, laboratory probes) make it easy to deploy and to use in any kind of environment such as small estuaries [1,2], river reaches [3, 4], single [5] and compound [6, 7] open-channels in laboratories.

\footnotetext{
${ }^{*}$ Corresponding author

Email address: yann.peltier90@gmail.com (Y. Peltier)
} 
Since the development of the ADV in the early 90's [8], numerous studies have been searching for the best ways to set-up the velocimeter parameters in order to get accurate measurements of velocity field in turbulent flows. Lane et al. [9] studied the impact of the sampling frequency, while McLelland and Nicholas [10] discussed the appropriate minimum required signal-to-noise ratio. Roy et al. [11], Chanson et al. [12] worked on the minimal recording length for a time-series depending on what turbulent parameter is considered (mean velocity signal, variance, triple-correlations). Meanwhile, Precht et al. [13], Dombroski and Crimaldi [14] focused their studies on the maximal distance relative to a solid boundary for which the ADV measurements collapse with those performed with a Laser Doppler Velocimeter. As pointed out by Chanson et al. [12], "Simply, raw, ADV velocity data are not true turbulence and should never be used without adequate post-processing". It is well known that the time-series of ADV measurements are polluted by various sources of noises that can be partially corrected [15]. The spikes due to aliasing of the Doppler signal can be corrected using the phase-space thresholding method of Goring and Nikora [16]. In a more general way, all the sources of noise (Doppler ambiguity, electronic circuitry's sampling errors, ...) entering as additional variance terms in the measured fluctuating velocity variances can be corrected using the methods proposed by Nikora and Goring [17], Voulgaris and Trowbridge [18], Hurther and Lemmin [15], Blankaert and Lemmin [19], Doroudian et al. [20].

Among all these studies, a lack of information can be however identified. In most studies dealing with ADV measurements or processing, the precision in the probe orientation is rarely mentioned. The comparison of the resulting error (uncertainty if the orientation is not well known) with the other sources of uncertainty justifying whether the data needs to be corrected is even more rare. Without these information, one cannot be sure that the presented experimental data-set is truly representative of the local physics. In the case of compound channel flows, for instance, this means that without a good confidence on the probe orientation, the measurements of the secondary currents of Prandtl's second kind [21, 22], so as the characterisation of the mixing process between the channels [7] cannot be validated.

As previously pointed out, studies specific to ADV dealing with probe misorientation are rare [23, 24]. Nevertheless, some studies dealing with misoriented electromagnetic current-meters exists [25, 26, 27, 28, 29, 30, 31] and their results can be partly transposed to ADV probes since their working principle are close. Heathershaw [29] estimated the error on the velocity measurements in the bottom boundary layer of a tidal current due to an electromagnetic current-meter misorientation. Roy et al. [30] analysed the effects of such misorientation on the vertical Reynolds shear stress in a gravel bed river and in a laboratory confluence. Trowbridge [31] proposed a method taking into account the surface-waves for measuring the Reynolds stress in the vicinity of shores.

The estimation of the error on the mean velocities due the ADV misorientation is straightforward using the classical matrix of rotation [32]. Similarly, the estimation of the error on the vertical Reynolds shear stress is well known $[29,30]$. By contrast, the estimation for the components of the Reynolds stress in the horizontal plane is not established. This paper then aims at evaluating the error on the horizontal components of the mean-velocity and of the Reynolds stress due to a misoriented ADV in the horizontal plane. The error due to the misorientation is first rigorously evaluated through theoretical considerations partly based on previous studies dealing with measurements 


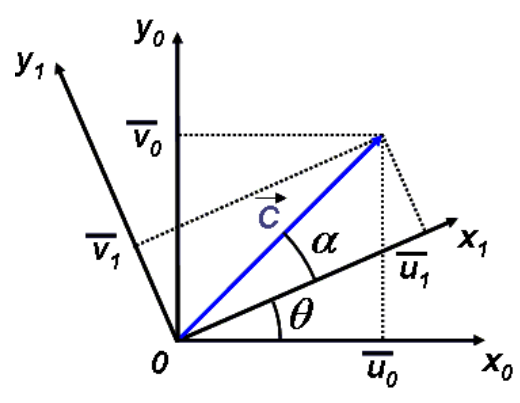

Figure 1: Illustration of the probe misorientation in the horizontal plane with respect to the reference frame aligned with the bank. The subscript "0" refers to the reference frame and the subscript "1" to the ADV coordinate frame.

on the vertical plane $[29,30]$. Second, these analytical relationships are used on experimental data measured under various flow conditions in a compound channel $[24,7]$. The experimental errors are then compared with those of more classical flows well detailed in the literature. Finally, these results are compared with the typical values of uncertainty due the various sources of noise.

\section{Theoretical background}

In the present paper, we use a Cartesian coordinate system in which $x, y$ and $z$ are respectively the longitudinal, the lateral and the vertical directions. Let $\left(0, x_{0}, y_{0}\right)$ be the reference frame attached to the experiment and $\left(0, x_{1}, y_{1}\right)$ be the ADV coordinate frame (Fig. 1), with $\theta$ the angle made by the ADV coordinate frame with the reference frame $(\theta>0$ in the counter-clockwise direction and $\theta=\theta(x, y)$, i.e. without misorientation in the vertical direction).

Let then $\left(U_{1}, V_{1}\right)$ be the instantaneous velocity components measured in the ADV coordinate frame and $C$ the norm of this velocity. In the reference frame, $C$ is obviously unchanged and the so-called "corrected velocity" components $\left(U_{0}, V_{0}\right)$ can be worked out as:

$$
\begin{aligned}
& U_{0}=U_{1} \cos \theta-V_{1} \sin \theta \\
& V_{0}=V_{1} \cos \theta+U_{1} \sin \theta
\end{aligned}
$$

Using the Reynolds decomposition $U=\bar{U}+u$ and $V=\bar{V}+v$ (over-bars: time-averaging, lowercase: fluctuation) and considering that $\overline{\bar{U}+u}=\bar{U}$, the mean velocities and the fluctuations can be separately rotated, which enables to work out the correlations used for calculating the following components of the horizontal Reynolds stress:

$$
\begin{aligned}
& \overline{u_{0}^{2}}=\overline{u_{1}^{2}} \cos ^{2} \theta+\overline{v_{1}^{2}} \sin ^{2} \theta-\overline{u_{1} v_{1}} \sin 2 \theta \\
& \overline{v_{0}^{2}}=\overline{v_{1}^{2}} \cos ^{2} \theta+\overline{u_{1}^{2}} \sin ^{2} \theta+\overline{u_{1} v_{1}} \sin 2 \theta \\
& \overline{u_{0} v_{0}}=\frac{\sin 2 \theta}{2}\left(\overline{u_{1}^{2}}-\overline{v_{1}^{2}}\right)+\overline{u_{1} v_{1}} \cos 2 \theta
\end{aligned}
$$

Notice that for transiting from the plane $\left(0, x_{0}, y_{0}\right)$ to the plane $\left(0, x_{1}, y_{1}\right)$ replace $\theta$ by $-\theta$ in Eqs. 1 and 2 . This transition can be seen as an other type of misorientation [30]. 


\subsection{Mean velocities}

In order to evaluate the error due to the ADV misalignment with the reference frame, a "fractional error" is analytically determined for each mean velocity component. It writes:

$$
\begin{aligned}
& E_{\bar{U}}=\frac{\overline{U_{1}}-\overline{U_{0}}}{\overline{U_{0}}}=\frac{\cos \alpha}{\cos (\alpha+\theta)}-1 \\
& E_{\bar{V}}=\frac{\overline{V_{1}}-\overline{V_{0}}}{\overline{V_{0}}}=\frac{\sin \alpha}{\sin (\alpha+\theta)}-1
\end{aligned}
$$

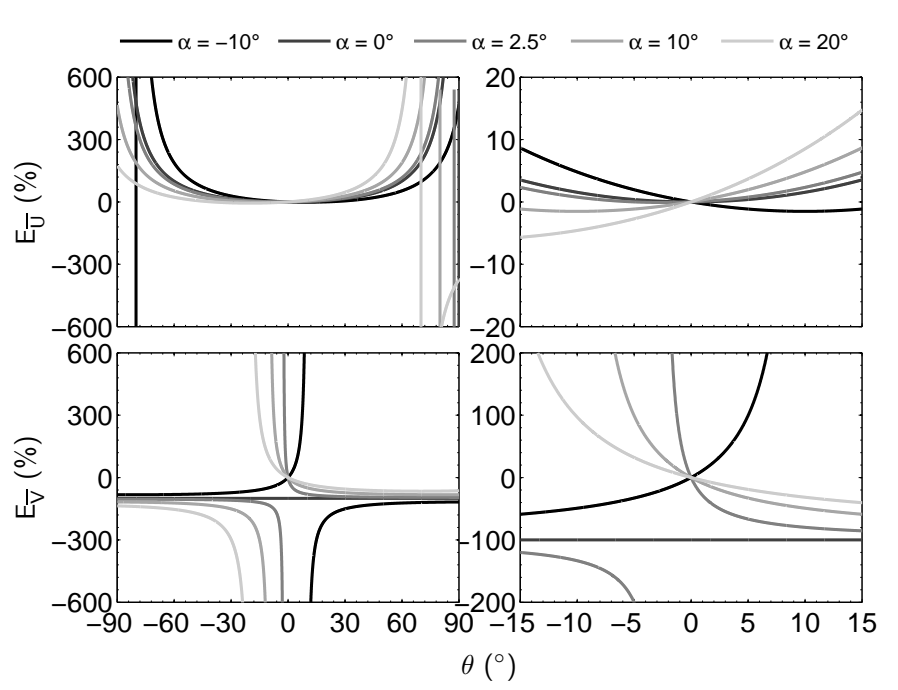

Figure 2: Fractional errors for the mean longitudinal velocity $E_{\bar{U}}$ and mean lateral velocity $E_{\bar{V}}$ with respect to $\theta$ for various angle $\alpha$. with $\alpha=\arctan \left(\overline{V_{1}} / \overline{U_{1}}\right)$ the angle between the streamline and the $\mathrm{x}$-wise axis of the ADV coordinate frame (see Fig. 1).

Plotting the fractional errors for $\theta$ in the range $\left[-90^{\circ} ; 90^{\circ}\right]$ (left figures in Fig. 2) reveals the respective evolutions of the two errors, exemplified by asymptotic behaviours. The fractional errors are $2 k \pi(k \in \mathbb{Z})$ periodic functions of $\alpha$ and $\theta$ and when they are plotted against $\theta$ for various positive angles $\alpha$ (Fig. 2), the following statements can be observed:

- For $\theta \in\left[-45^{\circ}-\alpha ; 45^{\circ}+\alpha\right], E_{\bar{U}}$ is at least one order of magnitude smaller than $E_{\bar{V}}$.

- For $\theta \in\left[-90^{\circ}-\alpha ; 90^{\circ}+\alpha\right], E_{\bar{U}}$ symmetrically evolves with respect to the axis passing by $\theta=-\alpha$. For this angle, the flow streamline is aligned with the reference frame and the fractional error reaches a local minimum equal to $E_{\bar{U}}=\cos \alpha-1$. By contrast, the fractional error is nil when $\theta=0$ or $\theta=-2 \alpha$.

- For $\theta \in\left[-90^{\circ}-\alpha ; 90^{\circ}+\alpha\right], E_{\bar{V}}$ asymptotically tends to infinity when $\theta=-\alpha$, i.e. when $E_{\bar{U}}$ reaches its local minimum. $E_{\bar{V}}$ also asymptotically tends to a local minimum (resp. maximum) when $\theta=-\alpha-90^{\circ}$ (resp. $\theta=-\alpha+90^{\circ}$ ) if $\alpha<0$ and $\theta=-\alpha+90^{\circ}$ (resp. $\theta=-\alpha-90^{\circ}$ ) if $\alpha>0$. Both local extrema coincide with the asymptotes to infinity observed in the upper plots in Fig. 2. 
Except if the beams of the ADV are inverted in the flow, realistic values are assumed to be rather in the range $\left[-2.5^{\circ} ; 2.5^{\circ}\right]$ and a focus is proposed in Fig. 2 (right).

\subsection{Reynolds stress}

Using Eqs. 2, the fractional errors are analytically determined for the horizontal components of the Reynolds stress:

$$
\begin{aligned}
& E_{\overline{u^{2}}}=\frac{\overline{u_{1}^{2}}-\overline{u_{0}^{2}}}{\overline{u_{0}^{2}}}=\left(\phi_{u v 0}^{-2}-1\right) \sin ^{2} \theta-\phi_{u v 0}^{-1} r_{u v 0} \sin (2 \theta) \\
& E_{\overline{v^{2}}}=\frac{\overline{v_{1}^{2}}-\overline{v_{0}^{2}}}{\overline{v_{0}^{2}}}=\left(\phi_{u v 0}^{2}-1\right) \sin ^{2} \theta+\phi_{u v 0} r_{u v 0} \sin (2 \theta) \\
& E_{\overline{u v}}=\frac{\overline{u_{1} v_{1}}-\overline{u_{0} v_{0}}}{\overline{u_{0} v_{0}}}=\cos (2 \theta)-1-\frac{\phi_{u v 0}^{-1}-\phi_{u v 0}}{2 r_{u v 0}} \sin (2 \theta)
\end{aligned}
$$

with $\phi_{u v 0}=\left(\overline{u_{0}^{2}} / \overline{v_{0}^{2}}\right)^{0.5}$ the ratio of $u_{0}$ and $v_{0}$ standard deviations and $r_{u v 0}=-\overline{u_{0} v_{0}} /\left(\overline{u_{0}^{2}} \overline{v_{0}^{2}}\right)^{0.5}$ the cross-correlation coefficient between $u_{0}$ and $v_{0}$. Notice that the third line of Eqs. 4 is analogous to the one proposed by Roy et al. [30] for the components of the fluctuating velocities, $(u, w)$, in the vertical plane.

Eqs. 4 are not easy to analyse because of the cosine and sine terms. Nevertheless, as previously observed, a more realistic values for $\theta$ is $\pm 2.5^{\circ}$. As a consequence, considering that $\theta=O(0)$ (i.e. $\left.|\tan \theta| \leq 0.1 \Leftrightarrow|\theta| \leq 5.71^{\circ}\right)$, Eqs. 4 can be linearised so that the fractional errors per degree write

$$
\begin{aligned}
\frac{E_{\overline{u^{2}}}}{\theta} & =\frac{\pi}{180}\left[\left(\phi_{u v 0}^{-2}-1\right) \theta-2 \phi_{u v 0}^{-1} r_{u v 0}\right] \\
\frac{E_{\overline{v^{2}}}}{\theta} & =\frac{\pi}{180}\left[\left(\phi_{u v 0}^{2}-1\right) \theta+2 \phi_{u v 0} r_{u v 0}\right] \\
\frac{E_{\overline{u v}}}{\theta} & =\frac{\pi}{180} \frac{\left(\phi_{u v 0}-\phi_{u v 0}^{-1}\right)}{r_{u v 0}}
\end{aligned}
$$

$E_{\overline{u^{2}}} / \theta$ and $E_{\overline{v^{2}}} / \theta$ (Eq. 5) are plotted with respect to $r_{u v 0}$ for various values of $\phi_{u v 0}$ and $\theta$ in Fig. 3. Both fractional errors are of the same order of magnitude. They both are almost equal to 5\%/deg in absolute value for a coefficient $r_{u v 0}= \pm 0.5$ with $\phi_{u v 0}=0.5$ for $E_{\overline{u^{2}}} / \theta$ and $\phi_{u v 0}=2.4$ for $E_{\overline{v^{2}}} / \theta\left(r_{u v 0} \in[-0.5 ; 0.5]\right.$ and $\phi_{u v 0} \in[0.5 ; 2.4]$ are typical values in open channel flows [32]). The fractional error per degree $E_{\overline{u^{2}}} / \theta$ decreases with increasing $\phi_{u v 0}$, while the contrary is observed for $E_{\overline{v^{2}}} / \theta$. Moreover, $\theta$ has little influence on the fractional error as shown in the right plots in Fig. 3.

As $E_{\overline{u v}} / \theta$ is independent from $\theta$ (see in Eq. 5), $E_{\overline{u v}} / \theta$ is plotted with respect to $r_{u v 0}$ for various values of $\phi_{u v 0}$ in Fig. 4. Unlike $\left|E_{\overline{u^{2}}} / \theta\right|$ and $\left|E_{\overline{v^{2}}} / \theta\right|,\left|E_{\overline{u v}} / \theta\right|$ decreases for increasing $\left|r_{u v 0}\right|$. In addition, $\left|E_{\overline{u v}} / \theta\right|>>\left|E_{\overline{u^{2}}} / \theta\right|,\left|E_{\overline{v^{2}}} / \theta\right|$ for near-zero values of $r_{u v 0}$ and they are equivalent when $r_{u v 0} \approx \pm 0.5$. 


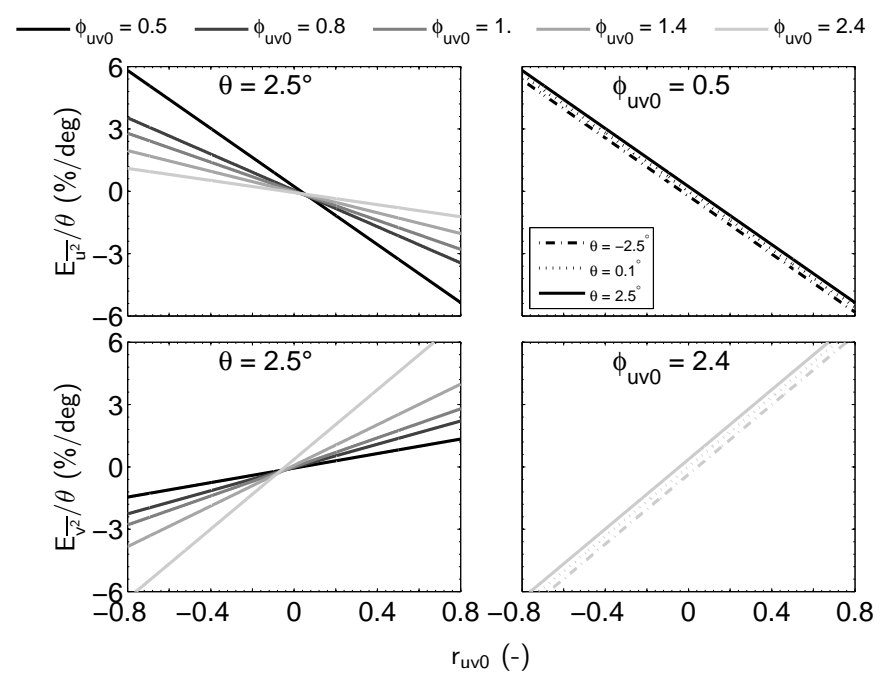

Figure 3: Fractional errors in percentage per degree for the auto-correlations $E_{\overline{u^{2}}} / \theta$ and $E_{\overline{v^{2}}} / \theta$ with respect to $\phi_{u v 0}, r_{u v 0}$ and $\theta$, considering that $\theta=O(0)$.

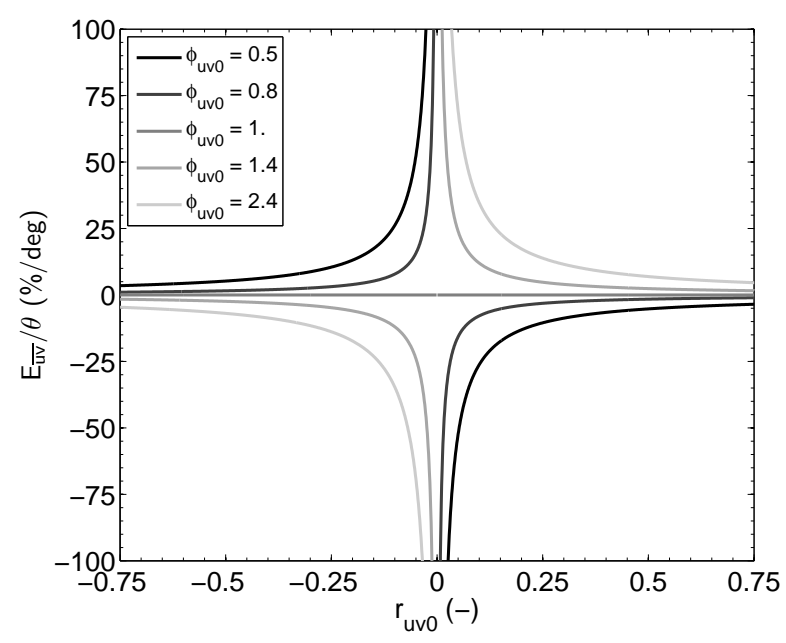

Figure 4: Fractional error in percentage per degree for the cross-correlation $E_{\overline{u v}} / \theta$ with respect to $\phi_{u v 0}$ and $r_{u v 0}$, considering that $\theta=O(0)$. 


\section{Experimental fractional error}

In the following section, the error due to device misorientation is evaluated through experimental data. The experimental fractional errors are worked out for a misalignment angle $\theta$ of $2.5^{\circ}$ using ADV measurements performed in a compound open-channel under various flow conditions. These errors are then compared with fractional errors calculated with data of more classical flows (boundary layer, mixing layer, plane jet...) for the same value of $\theta$.

\subsection{Case of compound channel flows}

\section{Set-up of the experiments}

Experiments were conducted at the Laboratoire de Mécanique des Fluides et d'Acoustique (LMFA, Lyon, France) in a $8 \mathrm{~m}$ long and $1.2 \mathrm{~m}$ wide straight asymmetric compound open-channel, with a longitudinal bed-slope of $0.18 \%$. The flume is PVC-made and its surface state is smooth. The main channel and the floopdlain cross-sections are rectangular (see in Fig. 5) and their widths are respectively equal to $0.4 \mathrm{~m}$ and $0.8 \mathrm{~m}$. The bank-full height in the main channel, $b$, is $5.1 \mathrm{~cm}$. As recommended by Bousmar et al. [33] for accelerating the flow establishment in a compound channel, separated filling tanks, inlets and downstream tailgates are used in the flume.
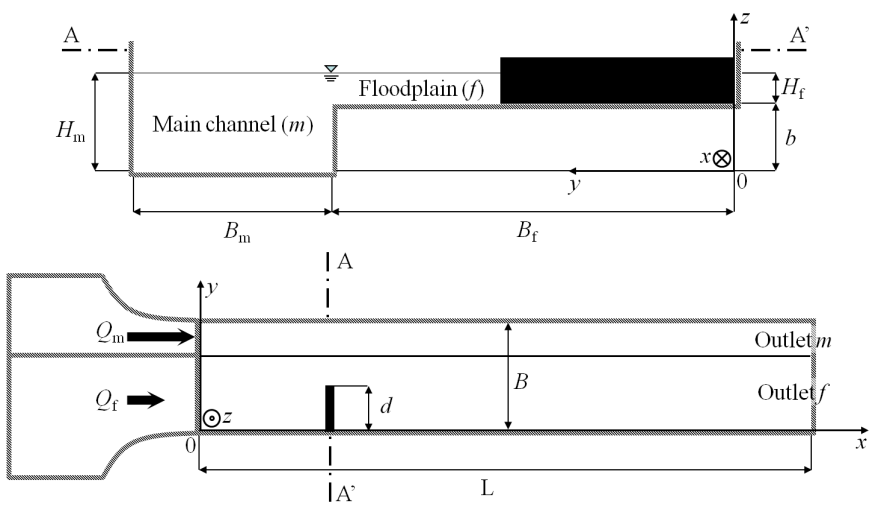

Figure 5: Definition sketch of the flume in the LMFA: cross-sectional and plan views (scheme is not to scale). The system origin is defined as: $x=0$ immediately downstream from the inlet tanks; $y=0$ on the lateral side-wall of the floodplain; $z=0$ on the bottom of the main channel.

The flow conditions of the data-set we use in this paper are described in Peltier [24], Peltier et al. [7] and include:

- Three uniform flow-cases, which relative flow depths, $H_{r}$, are close to $0.2,0.3$ and 0.4 in most part of the flume ( $H_{r}=H_{f} / H_{m}$, with $H_{f}$ the mean water depth in the floodplain and $H_{m}$ the mean water depth in the main channel respectively).

- Six non-uniform flow-cases with a thin obstacle on the floodplain, representing an embankment. The embankment was set at $x=2.5 \mathrm{~m}$ from the inlet, perpendicularly to the longitudinal direction (see in Fig. 5). Various lengths of embankment, $d$, were used, but the upstream discharge distributions and the tailgate settings were the same as used for the uniform flows. 


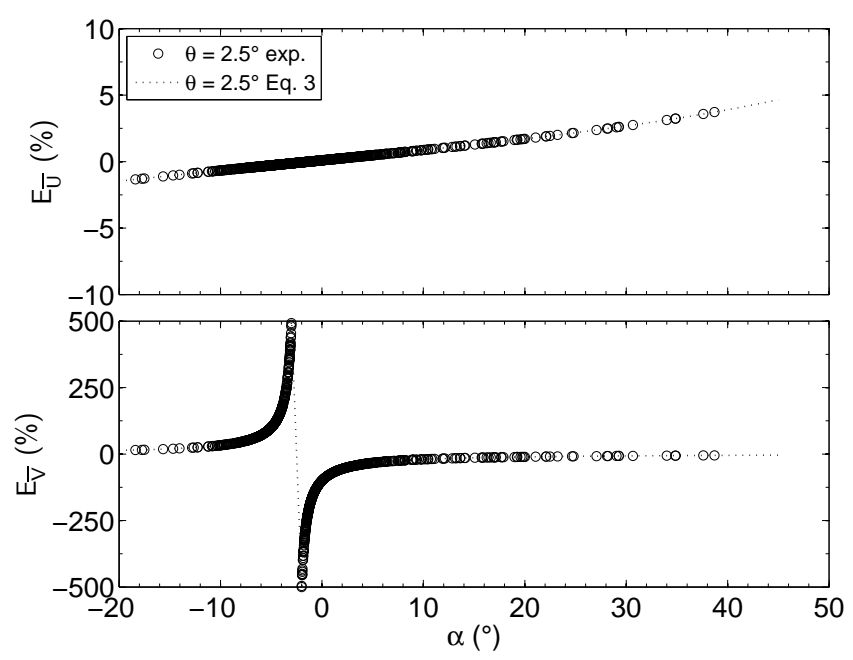

Figure 6: Experimental fractional errors for the mean velocities $E_{\bar{U}}$ and $E_{\bar{V}}$ with respect to $\theta$ and $\alpha$ with $\theta=2.5^{\circ}$.

The instantaneous velocities were measured using a 2D side-looking micro-ADV (Nortek, Vectrino ${ }^{+}$). The sampling frequency was set to $100 \mathrm{~Hz}$ with a signal-to-noise ratio greater than $20 \mathrm{~dB}$ in order to have weak influences of the noise [10]. The recording time was set at least to $3 \mathrm{~min}$ to ensure a good convergence of the mean and standard deviation of the signal (according to Chanson et al. [12], 18000 samples lead to an error of $\approx \pm 2 \%$ on the standard deviation). The measured velocity signals were finally despiked using the method of Goring and Nikora [16], but no specific method was applied for correcting the other sources of noise.

Due to the varied settings of the experiments, wide ranges of velocities $(C \in[0.1 ; 0.9] \mathrm{m} / \mathrm{s})$, of flow deviations $\left(\alpha \in\left[-30^{\circ} ; 20^{\circ}\right]\right)$, of cross-correlation coefficients $\left(r_{u v 0} \in[-0.57 ; 0.72]\right)$ and ratios of standard deviations $\left(\phi_{u v 0} \in\right.$ $[0.8 ; 3.8])$ were obtained.

\section{Fractional errors}

In order to estimate the error due to a misorientation of the ADV, we work out the fractional errors of this data-set (1) assuming that the measured data (the raw data) is well oriented in the reference frame $\left(0, x_{0}, y_{0}\right)$ and (2) imposing an artificial rotation of $\theta=2.5^{\circ}$ to this data to obtain the misoriented data in the ADV coordinate frame $\left(0, x_{1}, y_{1}\right)$. This angle of $2.5^{\circ}$ represents the maximal angle of misalignment by human eyes adjustment (above this angle, the misalignment can be easily detected).

The fractional errors are first calculated for the mean velocities using Eqs. 3. Both velocity components are independent from $C$ and $E_{\bar{U}}$ appears to be within $\pm 5 \%$, while $E_{\bar{V}}$ can reach several thousands of percent if $\alpha=-\theta=$ $-2.5^{\circ}$ (Fig. 6).

The fractional errors for the Reynolds stresses, calculated using Eqs. 4 are then plotted against the cross-correlation coefficient $r_{u v 0}$ and $\phi_{u v 0} \in[0.8 ; 2.4]$ in Fig. 7. The absolute value of $E_{\overline{u^{2}}}$ increases with increasing $\left|r_{u v 0}\right|$ and decreasing $\phi_{u v 0}$, while the absolute value of $E_{\overline{v^{2}}}$ increases with both increasing $\left|r_{u v 0}\right|$ and $\phi_{u v 0}$. Nevertheless these fractional errors 


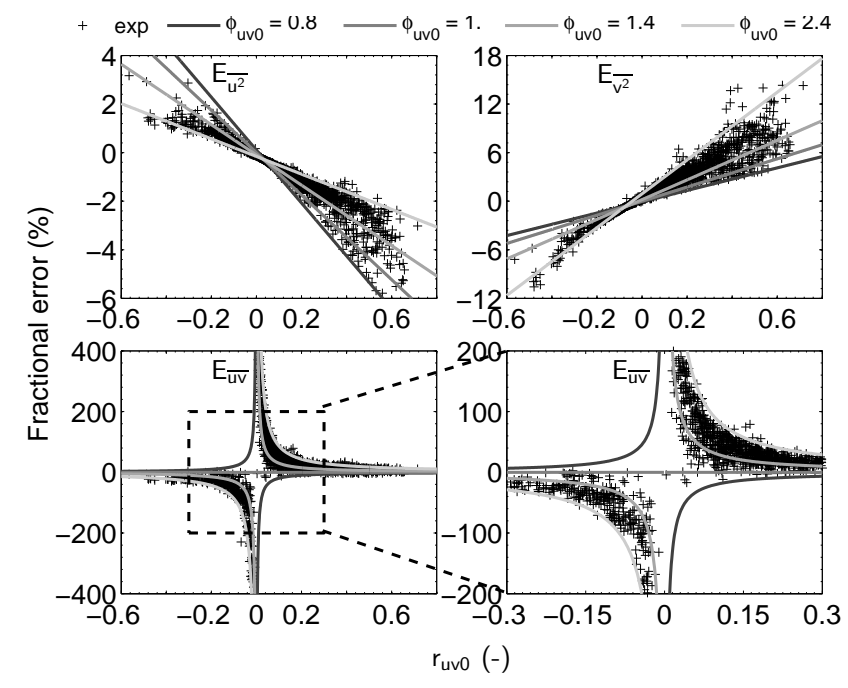

Figure 7: Experimental fractional errors for the Reynolds stresses $E_{\overline{u^{2}}}, E_{\overline{v^{2}}}$ and $E_{\overline{u v}}$ with respect to $r_{u v 0}$ for $\phi_{u v 0} \in[0.8 ; 2.4]$ with $\theta=2.5^{\circ}$.

remain relatively low. $E_{\overline{u^{2}}}$ ranges from $-5.8 \%$ to $3.2 \%$, while $E_{\overline{v^{2}}}$ ranges from $-10.6 \%$ to $14.3 \%$. By contrast, the absolute value of $E_{\overline{u v}}$ can reach several hundreds of percent when $\left|r_{u v 0}\right|$ goes to 0 . In the mixing layer of the compound channel, typical values of $\left|r_{u v 0}\right|$ are greater than 0.2 and $\left|E_{\overline{u v}}\right|$ is therefore lower than $90 \%$.

The fractional errors $E_{\overline{u^{2}}}, E_{\overline{v^{2}}}$ and $E_{\overline{u v}}$ are plotted in Fig. 8 relative to the corresponding values of the Reynolds stresses measured in the reference frame. While no tendency is observed for $-\rho \overline{u^{2}}, E_{\overline{v^{2}}}$ decreases with decreasing $-\rho \overline{v^{2}}$, which could indicate that slow flow motions are more prone to orientation imprecisions. Concerning the Reynolds shear stress $-\rho \overline{u v}, E_{\overline{u v}}$ is relatively high $\left(\left|E_{\overline{u v}}\right| \geq 10 \%\right)$ when $-\rho \overline{u v}$ is smaller than $1 \mathrm{~Pa}$ in absolute. Beyond $1 \mathrm{~Pa}$, the fractional error is within $\pm 20 \%$. This result is of importance for the study of shear flows in compound open-channels: in areas of high Reynolds shear stress, we can ensure relatively low error due to a misorientation of the ADV. By contrast, in the areas, where $|-\rho \overline{u v}|$ is smaller than $1 \mathrm{~Pa}$, the error can be prohibitive. The accurate measurement of the Reynolds stresses in the horizontal plane is indeed paramount for validating a turbulent model (notably for $2 \mathrm{D}$ depth-averaged models) and an error of several tens (or hundreds) of percent is unacceptable.

\subsection{Comparison with more classical flows}

In the previous sub-section, we showed that the fractional errors for the mean velocities only depends on $\theta$ and $\alpha$ and is therefore independent from the type of the flow. By contrast, the fractional errors for the Reynolds stresses are conditioned by the turbulent characteristics of the flow and can be greater than several hundreds of percent, especially when considering the Reynolds shear stress $-\rho \overline{u v}$. Nevertheless, compound channel flows are quite particular flows and their fractional error values could be specific to this kind of flow. In the literature, the turbulent characteristics of classical flows are well detailed, which allow us to calculate typical fractional errors to be compared with the ones of the present experiments (see Tabs. 1 and 2). The computed fractional errors correspond to hypothetical errors if the measurements would have been performed using an ADV with a $2.5^{\circ}$ misalignment angle. 


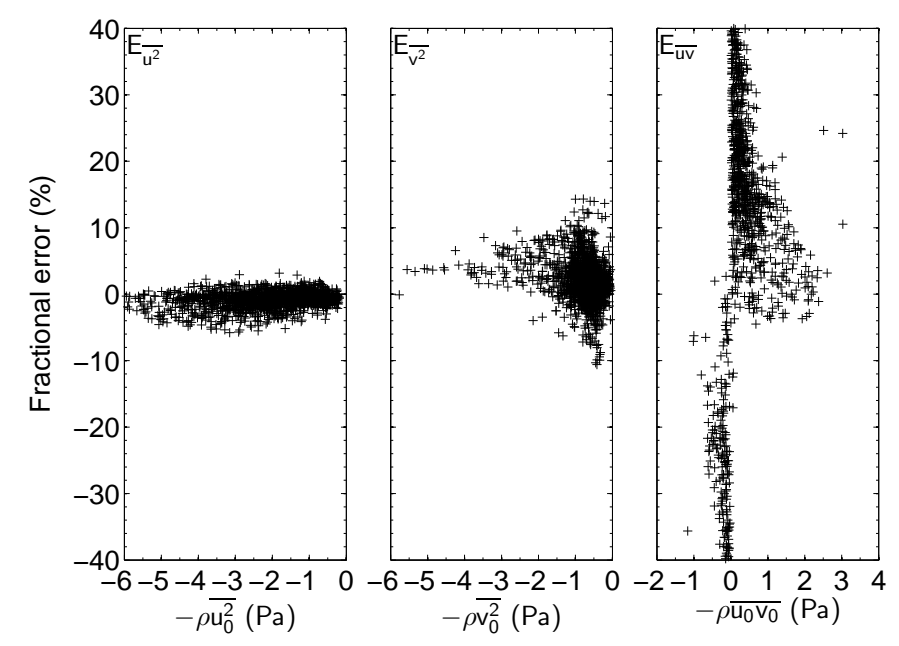

Figure 8: Experimental fractional errors $\left(E_{\overline{u^{2}}}, E_{\overline{v^{2}}}, E_{\overline{u v}}\right)$ plotted with respect to the corresponding values of Reynolds stresses $\left(-\rho \overline{u_{0}^{2}},-\rho \overline{v_{0}^{2}},-\rho \overline{u_{0} v_{0}}\right)$ for $\theta=2.5^{\circ}$.

Table 1: Fractional errors for flows with a mean velocity gradient mainly occurring in the horizontal plane. Calculation with $\theta=2.5^{\circ}$.

\begin{tabular}{lcccccc}
\hline Type of flow & Authors & $\phi_{u v_{0}}(-)$ & $r_{u v_{0}}(-)$ & $E_{\overline{u^{2}}}(\%)$ & $E_{\overline{v^{2}}}(\%)$ & $E_{\overline{u v}}(\%)$ \\
\hline Compound channel flows & Present data & {$[0.8 ; 3.8]$} & {$[-0.57 ; 0.72]$} & {$[-5.8 ; 3.2]$} & {$[-10.6 ; 14.3]$} & $>100$ \\
\hline Mixing layer: & & & & & & \\
- Centre & {$[34]$} & {$[1.2 ; 1.6]$} & {$[0.42 ; 0.62]$} & {$[-4 ;-2]$} & {$[6 ; 7]$} & {$[3 ; 10]$} \\
- Side & & {$[0.8 ; 1.8]$} & {$[0.14 ; 0.5]$} & {$[-3 ; 0.8]$} & {$[2 ; 5]$} & {$[3 ; 30]$} \\
\hline Plane Jet: & & & & & & \\
- Near the centreline & {$[35]$} & 1.6 & -0.42 & 2.1 & -5.6 & -10.4 \\
- Near the edges & & 1.1 & -0.22 & 1.6 & -2.1 & -4.7 \\
\hline Homogeneous shear flows & {$[36]$} & 1.67 & 0.45 & -2.4 & 6.9 & 10 \\
$(x / B=7.5)$ & & & & & & \\
\hline
\end{tabular}


Table 2: Fractional errors for flows with a mean velocity gradient mainly occurring in the vertical plane. Calculation with $\theta=2.5^{\circ}$. $E_{\overline{u w}}$ is calculated using the formula proposed by Roy et al. [30].

\begin{tabular}{lcccc}
\hline Type of flow & Authors & $\phi_{u w_{0}}(-)$ & $r_{u w_{0}}(-)$ & $E_{\overline{u w}}(\%)$ \\
\hline $\begin{array}{l}\text { Smooth boundary layer: } \\
\text { Viscous sub-layer }\end{array}$ & {$[14]$} & {$[3.4 ; 6]$} & {$[0.19 ; 0.34]$} & {$[63 ; 110]$} \\
Log-layer and outer layer & & {$[0.8 ; 4]$} & {$[0.03 ; 0.4]$} & {$[-0.3 ; 50]$} \\
\hline Transition boundary layer: & {$[37]$} & {$[2.3 ; 3.9]$} & {$[0.47 ; 0.52]$} & {$[15 ; 33]$} \\
Near the bottom & & {$[1.7 ; 2]$} & {$[0.38 ; 0.53]$} & {$[10 ; 12]$} \\
In the bulk & & & & \\
\hline Fully rough boundary layer: & {$[38,39]$} & {$[3.1 ; 4.1]$} & {$[0.13 ; 0.46]$} & {$[25 ; 122]$} \\
Near the bottom & & {$[2.2 ; 2.9]$} & {$[0.41 ; 0.5]$} & {$[16 ; 22]$} \\
In the bulk & {$[29]$} & {$[1.5 ; 2.5]$} & {$[0.07 ; 0.25]$} & {$[18 ; 151]$} \\
\hline Boundary layer in a tidal current & & & &
\end{tabular}

The parameters $\phi_{u v 0}$ and $r_{u v 0}$ were first determined for three types of flow for which the mean velocity gradient mainly occurs in the horizontal plane (Tab. 1): mixing layer created by a splitter plate at the flume entrance [34], plane jet [35] and homogeneous shear flow [36]. It results that $\phi_{u v 0}$ and $r_{u v 0}$ for the three cases are within the ranges of the compound channel flow, which leads to fractional errors of the same order of magnitude. Similarly to the compound channel flows, the fractional errors $E_{\overline{u^{2}}}$ and $E_{\overline{v^{2}}}$ increase with the Reynolds shear stress magnitude, while $E_{\overline{u v}}$ is minimal in the zone of intense shear, where it can still reach $10 \%$.

Although, the main aims of this paper was to assess the error due to a misorientation of an ADV with respect to the horizontal plane, we also compare these errors with the ones on the vertical plane from data in the literature. As pointed out in section 2, by replacing $v$ by $w$ (the vertical fluctuating velocity) in the third line of Eqs. 4, Roy et al. [30] found an expression for $E_{\overline{u w}}$ similar to the one of $E_{\overline{u v}}$, but none for $E_{\overline{u^{2}}}$ and $E_{\overline{w^{2}}}$. In Tab. 2, only $E_{\overline{u w}}$ is therefore presented.

The parameters $\phi_{u w 0}$ and $r_{u w 0}$ in Tab. 2 were determined with data of four different boundary layers with a mean velocity gradient mainly occurring in the vertical plane. The ranges of $\phi_{u w 0}$ and $r_{u w 0}$ are similar to those of $\phi_{u v 0}$ and $r_{u v 0}$ in Tab. 1. The magnitude of $E_{\overline{u w}}$ is also similar to $E_{\overline{u v}}$, the greater errors due to a misorientation being located in the viscous sub-layer of the smooth boundary layer [14] or in the roughness layer of the transitional [37] and of the fully rough boundary layers [38, 39].

\section{Discussion}

In the previous section, we showed that the error due to the ADV misorientation can be very high, especially when estimating the Reynolds shear stress components. This section now aims at comparing this error with the classical 
sources of uncertainty generally considered in ADV measurements.

According to Hurther and Lemmin [15], any deployment errors aside, six sources of noise affect the turbulence measurements using an ADV:

1. the Doppler ambiguity at high frequency

2. the effects of the mean flow shear stress present within the sampling volume

3. the phase distortion of the emitted front wave

4. the electronic circuitries

5. the effects of the turbulent structures smaller or of the same size as the sampling volume

6. the spatial averaging of the instantaneous velocity field in the sampling volume.

Among these six sources, the five first ones can be corrected by applying the methods described in Hurther and Lemmin [15], Blankaert and Lemmin [19] for an Acoustic Doppler Velocimeter Profiler. The remaining source (the sixth) is a problem inherent to the geometry of the device and cannot be corrected. These sources of noise mainly affect the normal Reynolds stress [15]. By contrast, the Reynolds shear stresses are theoretically independent from the five first sources, but the spatial averaging within the sampling volume (the sixth source) can be responsible for large uncertainties, especially if the measurements are performed close to irregular boundaries (gravel-bed) or obstacles (embankment).

According to Hurther and Lemmin [15], in a flow over a gravel bed, the uncertainty due to the sum of the six sources of noise can reach $\approx 5 \%$ for the normal Reynolds stresses and the Reynolds shear stress in absence of irregular boundaries and obstacles. In the "pertubated" zones this uncertainty is much higher. Hurther and Lemmin [15] indeed showed that in the rough sub-layer, the uncertainty on the Reynolds shear stress can reach $120 \%$. These orders of magnitude for this uncertainty are similar to the error due to a misorientation of the probe in the flow (Tabs 1 and 2). As a consequence, both error and uncertainty should then be taken into account.

In the paper, we have considered that errors due to the probe misorientation and uncertainties listed above were independent from each other. This is actually not the case. Indeed, the error due to the probe misorientation depends on the quality of the velocity measurement. As shown in Figs. 3 and 4, if $r_{u v 0}$ and $\phi_{u v 0}$ values are badly estimated due to uncertainties, the errors induced by the probe misorientation can be strongly affected. For instance, a change in $60 \%$ of $\phi_{u v 0}$ for high $r_{u v 0}$ induces a change of $38 \%$ in $E_{\overline{u^{2}}} / \theta$ and a change of $70 \%$ for $E_{\overline{u v}} / \theta$.

\section{Conclusion}

The error due to a misorientation of an Acoustic Doppler Velocimeter in the horizontal plane was estimated on the horizontal components of the mean velocity and of the Reynolds stresses, first analytically and then using an experimental data-set of compound channel flows with a misalignment angle of $2.5^{\circ}$. The following conclusions are drawn: 
1. The fractional error for the mean longitudinal velocity is never greater than $|5| \%$.

2. The fractional error for the lateral mean velocity can be several order greater, especially when the misalignment angle is equal to the opposite angle between the velocity direction and the x-axis of the ADV coordinate frame.

3. The absolute fractional errors for the normal Reynolds stresses $-\rho \overline{u^{2}}$ and $-\rho \overline{v^{2}}$ do not exceed $5.8 \%$ and $14.3 \%$ respectively.

4. The absolute fractional error for the Reynolds shear stress $-\rho \overline{u v}$ increases when the absolute value of the coefficient correlation $r_{u v 0}$ decreases. The fractional error can reach several thousand percent at low $r_{u v 0}$. Nevertheless, in the zones of high velocity gradient, where $-\rho \overline{u v} \geq 1 \mathrm{~Pa}$ or $-\rho \overline{u v} \leq-1 \mathrm{~Pa}$, the fractional error does not exceed $\pm 20 \%$.

The fractional errors worked out with the compound channel flows were then compared with the ones of classical flows from the literature. The error appears to be of the same order of magnitude as the compound channel flows.

We finally discussed the necessity of taking into account the ADV misorientation for assessing the uncertainty on the ADV measurements. Using the numerous studies dealing with noise cancellation and uncertainty estimation due to the various sources of noise, we highlighted that the error due to the misorientation is of the same order of magnitude as the uncertainty due to the noises. As a result, they should both be used by ADV users in order to interpret their measurements, especially when working with the components of the Reynolds shear stress.

ADV users must keep in mind that even if the error on the ADV orientation can be high, such a device is a powerful tool to measure good turbulent characteristics of flows in which more "accurate" measurement methods could not be used. Moreover, these errors can be reduced by attentively deploying the device in the flume or in the field or by applying an adequate correction on the data during post-processing. In the literature, one can find studies dealing with correction methods for ADV measurements $[3,5,18]$. However, as pointed out by several authors, unless misalignment angles of each sampling station with respect to the reference frame are known, most of these methods can only correct for a constant misalignment angle across the entire section. To the authors knowledge, a simple way to correct one sampling location at the time must still be found.

\section{Acknowledgment}

The research was funded by Irstea and by the Rhône-Alpes region (SRESR - EXPLORA'DOC 2008 Bourse Cluster 6). The authors are grateful to F. Thollet and M. Lagouy for their technical support.

\section{References}

[1] Voulgaris G, Meyers ST. Temporal variability of hydrodynamics, sediment concentration and sediment settling velocity in a tidal creek. Cont Shelf Res 2004;24:1659-83.

[2] Chanson H, Trevethan M, Aoki SI. Acoustic Doppler velocimetry (ADV) in small estuary: Field experience and signal post-processing. Flow Meas Inst 2008;19:307-13. 
[3] Dietrich WE, Smith JD. Influence of point bar on flow trough curved channels. Water Resour Res 1983;19(5):1173-92.

[4] Strom KB, Papanicolaou AN. ADV measurements around a cluster microform in a shallow mountain stream. J Hydraulic Eng, ASCE 2007;133(12):1379-89. doi:10.1061/(ASCE)0733-9429(2007)133:12(1379).

[5] Lacey RWJ, Rennie CD. Laboratory investigation of turbulent flow structure around a bed-mounted cube at multiple flow stages. J Hydraulic Eng, ASCE 2012;138(1):71-84

[6] Proust S, Peltier Y, Fernandes J, Leal J, Thollet F, Lagouy M, et al. Effect of different inlet flow conditions on turbulence in a straight compound open channel. In: 34th IAHR Congr.: Balance and Uncertainty; Water in a Changing World, 26 June-01 July 2011 , Brisbane, Australia. 2011, p. 3714-21.

[7] Peltier Y, Proust S, Rivière N, Paquier A, Shiono K. Turbulent flows in straight compound open-channel with a transverse embankment on the floodplain. J Hydraulic Res 2013. doi:10.1080/00221686.2013.796499.

[8] Lohrmann A, Cabrera R, Kraus NC. Acoustic Doppler Velocimeter (ADV) for laboratory use. In: Reprinted from Fundamentals and Advancements in Hydraulic Measurements and Experimentation, 1-5 August 1994, Buffalo, New-York, USA. 1994 , p. $351-65$.

[9] Lane SN, Biron PM, Bradbrook KF, B.Butler J, Chandler JH, Crowell MD, et al. Three dimensional measurement of river channel flow processes using Acoustic Doppler Velocimetry. Earth Surf Proc Landforms 1998;23:1247-67.

[10] McLelland SJ, Nicholas AP. A new method for evaluating errors in high-frequency ADV measurements. Hydrological Proc 2000;14:351-66.

[11] Roy AG, Buffin-Belanger T, Lamarre H, Kirkbride AD. Size, shape and dynamics of large scale turbulent flow structures in a gravel-bed river. J Fluid Mech 2004;500:1-27.

[12] Chanson H, Trevethan M, Koch C. Discussion of turbulence measurements with acoustic Doppler velocimeters by C. M. Garcìa, M. I. Cantero, Y. Niño and M. H. Garcìa. J Hydraulic Eng, ASCE 2007;133(11):1283-6.

[13] Precht E, Janssen F, Huettel M. Near-bottom performance of the Acoustic Doppler Velocimeter (ADV) - a comparative study. Aqua Eco 2006;40:481-92.

[14] Dombroski DE, Crimaldi JP. The accuracy of acoustic Doppler velocimetry measurements in turbulent boundary layer flows over a smooth bed. Limnology Oceanogr Methods 2007;5(1):23-33.

[15] Hurther D, Lemmin U. A correction method for turbulence measurements with a 3D acoustic doppler velocity profiler. J Atmos Oceanic Tech, AMS 2001;18:446-58.

[16] Goring DG, Nikora VI. Despiking Acoustic Doppler Velocimeter data. J Hydraulic Eng, ASCE 2002;128(1):117-26.

[17] Nikora VI, Goring DG. ADV measurements of turbulence: can we improve their interpretation. J Hydraulic Eng, ASCE 1998;124(6):630-4.

[18] Voulgaris G, Trowbridge JH. Evaluation of the Acoustic Doppler Velocimeter (ADV) for turbulence measurements. J Atmos Oceanic Tech, AMS 1998;15:272-89.

[19] Blankaert K, Lemmin U. Means of noise reduction in acoustic turbulence measurements. J Hydraulic Res 2006;44(1):3-17.

[20] Doroudian B, Hurther D, Lemmin U. Discussion of turbulence measurements with acoustic Doppler velocimeters by C. M. Garcìa, M. I. Cantero, Y. Niño and M. H. Garcìa. J Hydraulic Eng 2007;133(11):1286-9.

[21] Tominaga A, Nezu I. Turbulent structure in compound open channel flow. J Hydraulic Eng, ASCE 1991;117(1):21-41.

[22] Shiono K, Feng T. Turbulence measurements of dye concentration and effects of secondary flow on distribution in open channel flows. J Hydraulic Eng, ASCE 2003;129 (5):373-84.

[23] Snyder WH, Castro IP. Acoustic Doppler velocimeter evaluation in stratified towing tank. J Hydraulic Eng, ASCE 1999;125(6):595-603.

[24] Peltier Y. Physical modelling of overbank flows with a groyne set on the floodplain. Ph.D. thesis; Université de Lyon; Lyon, France; 2011.

[25] Deacon EL. The levelling error in Reynolds stress measurement. Bull Am Meteor Soc 1968;49:836.

[26] Pond S. Some effects of buoy motion on measurements of wind speed and stress. J Geophys Res 1968;73:507-12.

[27] Kaimal JC, Haugen DA. Some errors in the measurement of Reynolds stress. J App Meteor 1969;8:460-2.

[28] Pond S, Phelps GT, Paquin JE, McBean G, Stewart RW. Measurements of the turbulent fluxes of the momentum, moisture and sensible heat over the ocean. J Atmos Sci 1971;28:901-17.

[29] Heathershaw AD. The turbulent structure of the bottom boundary layer in a tidal current. Goeophys J R astr SOC 1979;58:395-430. 
[30] Roy AG, Biron P, de Serres B. On the necessity of applying a rotation to instantaneous velocity measurements in river flows. Earth Surf Proc Landforms 1996;21:817-27.

[31] Trowbridge JH. On a technique for measurement of turbulent shear stress in the presence of surface waves. J Atmos Oceanic Tech, AMS $1998 ; 15: 290-8$.

[32] Nezu I, Nakagawa H. IAHR monograph series: Turbulence in open-channel flows. A. A. Balkema Publishers, Rotterdam, The Netherlands; 1993.

[33] Bousmar D, Rivière N, Proust S, Paquier A, Morel R, Zech Y. Upstream discharge distribution in compound-channel flumes. J Hydraulic Eng, ASCE 2005;131:408-12.

[34] Uijttewaal WSJ, Booij R. Effects of shallowness on the development of free-surface mixing layers. Phy Fluids 2000;12:392-402.

[35] Heskestad G. Hot-wire measurements in a plane turbulent jet. J Appl Mech 1965;32:721-34.

[36] Tavoularis S, Corrsin S. Experiments in nearly homogeneous turbulent shear flow with a uniform mean temperature gradient. Part I. J Fluid Mech 1981;104:311-47.

[37] Bigillon F, no YN, Garcia MH. Measurements of turbulence characteristics in an open-channel flow over a transitionally-rough bed using particle image velocimetry. Exp Fluids 2006;41(6):857-67. doi:10.1007/s00348-006-0201-2.

[38] Mignot E, Barthélémy E, Hurther D. Double-averaging analysis and local flow characterization of near-bed turbulence in gravel-bed channel flows. J Fluid Mech 2009;618:279-303.

[39] Dey S, Das R. Gravel-bed hydrodynamics: double-averaging approach. J Hydraulic Eng 2012;138(8):707-25. doi:10.1061/(ASCE)HY.19437900.0000554 
The following symbols are used in this paper:

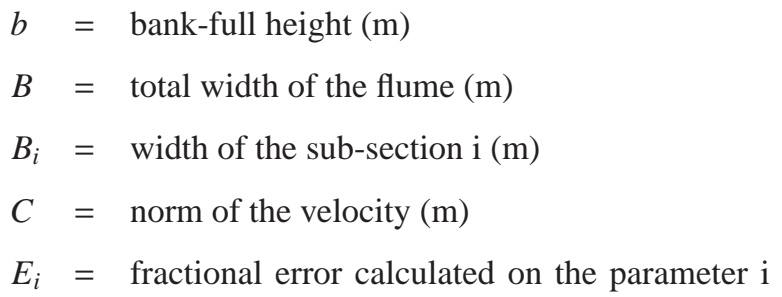

$(-)$

$h=$ local water depth $(\mathrm{m})$

$H_{i}=$ water depth in the sub-section $\mathrm{i}(\mathrm{m})$

$H_{r}=$ relative flow depth (-)

$L=$ length of the flume $(\mathrm{m})$

$r_{u v}=$ correlation coefficient of $u$ and $v(-)$

$r_{u w}=$ correlation coefficient of $u$ and $w(-)$

$Q_{i}=$ discharge in the sub-section $\mathrm{i}\left(\mathrm{m}^{3} / \mathrm{s}\right)$

$U=$ instantaneous longitudinal velocity $(\mathrm{m} / \mathrm{s})$

$\bar{U}=$ mean longitudinal velocity $(\mathrm{m} / \mathrm{s})$

$u=$ longitudinal fluctuating velocity $(\mathrm{m} / \mathrm{s})$

$V=$ instantaneous lateral velocity $(\mathrm{m} / \mathrm{s})$

$\bar{V}=$ mean lateral velocity $(\mathrm{m} / \mathrm{s})$

$v=$ lateral fluctuating velocity $(\mathrm{m} / \mathrm{s})$

$w=$ vertical fluctuating velocity $(\mathrm{m} / \mathrm{s})$

$x=$ streamwise direction $(\mathrm{m})$

$y=$ spanwise direction $(\mathrm{m})$

$z=$ vertical direction $(\mathrm{m})$

$\alpha=$ angle of the streamline velocity with respect to the $\mathrm{x}$-axis of the ADV coordinate frame $\left(^{\circ}\right)$

$\phi_{u v}=$ ratio of the standard deviation of $u$ by the one of $v$

$\phi_{u w}=$ ratio of the standard deviation of $u$ by the one of $w$

$\theta=$ angle between the reference frame and the ADV coordinate frame $\left(^{\circ}\right)$

$\rho \quad=\quad$ water density $\left(\mathrm{Kg} / \mathrm{m}^{3}\right)$ 
The following subscripts are used in this paper:

$0=$ refers to the reference frame

$1=$ refers to the ADV coordinate frame

$f \quad=$ floodplain

$m \quad=$ main channel 